\title{
Detector Performances Studies at Muon Collider
}

\section{Donatella Lucchesi*}

University and INFN of Padova, Padova, Italy

\section{Nazar Bartosik, Nadia Pastrone}

INFN Sezione di Torino, Torino, Italy

\section{Alessandro Bertolin, Alessio Gianelle, Lorenzo Sestini \\ INFN Sezione di Padova, Padova, Italy}

\section{Massimo Casarsa}

INFN Sezione di Trieste, Trieste, Italy

\section{Francesco Collamati}

INFN Sezione di Roma, Roma, Italy

\section{Alfredo Ferrari}

CERN, Geneva, Switzerland

\section{Anna Ferrari}

HZDR, Dresden, Germany

\section{Nikolai Mokhov, Sergei Striganov}

Fermilab, Batavia, Illinois, U.S.A

\section{Paola Sala}

INFN Sezione di Milano, Milano, Italy

The Muon Collider is a possible option for the next generation of high energy collider machines. It would permit to achieve the energy frontier in lepton collisions, without occurring in significant synchrotron radiation losses as in electrons rings. Among the technological challenges in the realization of such a machine, the treatment of the beam-induced background is one of the most critical issues for the detectors. Beams with intensity of the order of $10^{12}$ muons per bunch are necessary to obtain the desired luminosity, therefore the muons decay rate is very high. Beam decay products and subsequent particles from secondary interactions with the machine elements can reach the interaction point, limiting the physical performance of the detector. A study of the beam-induced background is presented together with possible strategies for its reduction. Preliminary results on the reconstruction of a benchmark process, $\mu^{+} \mu^{-} \rightarrow H v \bar{v} \rightarrow b \bar{b} v \bar{v}$, including the beam-induced background are illustrated as demonstration of physics measurements feasibility in this harsh environment.

European Physical Society Conference on High Energy Physics - EPS-HEP2019 -

10-17 July, 2019 Ghent, Belgium

\footnotetext{
*Speaker.
} 


\section{Motivation for a muon collider}

The Standard Model, the guide for physicists in the last fifty years, can not predict what to investigate to go beyond its predictions and answer the open questions. The energy where the so called New Physics can manifest itself could be very far from what any collider can achieve. The Higgs boson offers an important tool to investigate physics beyond the Standard Model: the accurate reconstruction of the shape of the Higgs potential. The Standard Model predicts a shape

$$
V=\frac{1}{2} m_{h}^{2} h^{2}+\left(1+k_{3}\right) \lambda_{h h h}^{S M} v h^{3}+\left(1+k_{4}\right) \lambda_{h h h h}^{S M} h^{4}
$$

with a range of values expected for $k_{3}$ and $k_{4}$ that have to be measured experimentally. Among the future colliders proposed so far, the muon collider is the only one that allows to produce enough double and triple Higgs events to measure directly $k_{3}$ and $k_{4}$ [1].In fact, a muon collider can reach energies in the center-of-mass higher than any electron-positron collider thanks to the minimal synchrotron and beamsstrhalung radiation. Preliminary muon collider designs exist, which are being studied by different groups as summarized in the input from the muon collider working group to the European strategy update [2]. Here, detector challenges due to the muon decay are presented.

\section{Beam-induced background}

The muon decay products generate a stream of secondary and tertiary particles that arrive to the interaction region (IR) posing a serious issue to physics measurements. The exact rate and composition of the beam-induced background depend on the beam energy, the layout of the accelerator around the interaction point (IP), the experimental hall, and the detector itself. The study of this background has been performed by the MAP collaboration [3] and resulted in a specific Machine-Detector-Interface (MDI) for two, at the moment, collider center-of-mass energies [4, 5]. The proposed solution consists of a pair of cone-shaped tungsten shields (nozzles) in the vicinity of the IP with the cone angle optimized depending on the beam energy and IR lattice. The results presented here use the beam-induced background generated by using the MARS15 code [6] for $1.5 \mathrm{TeV}$ center-of-mass energy collider. Figure 1 (left) illustrates the IR with the detector, the nozzles and the tracks of the beam-induced background as simulated by MARS15, and (right) the particle composition arriving to the detector for a single bunch crossing. The beam-induced background particles are characterized by low momentum, as shown in Figure 2 (left) for example the secondary and tertiary charged hadrons have an average momentum of the order of $500 \mathrm{MeV}$. Another distinctive feature of the background particles from muon decays is represented by their timing. Figure 2 (right) shows the distributions of the time of arrival at the detector entry point with respect to the bunch crossing time for the different background components. For each particle type the distribution has a long tail and central Gaussian-like shape except the neutrons.

\section{Detector performance study}

The characteristics of the beam-induced background were not fully exploited to minimize it in the detector design used for the previous studies, in fact the detector model is inherited from the 

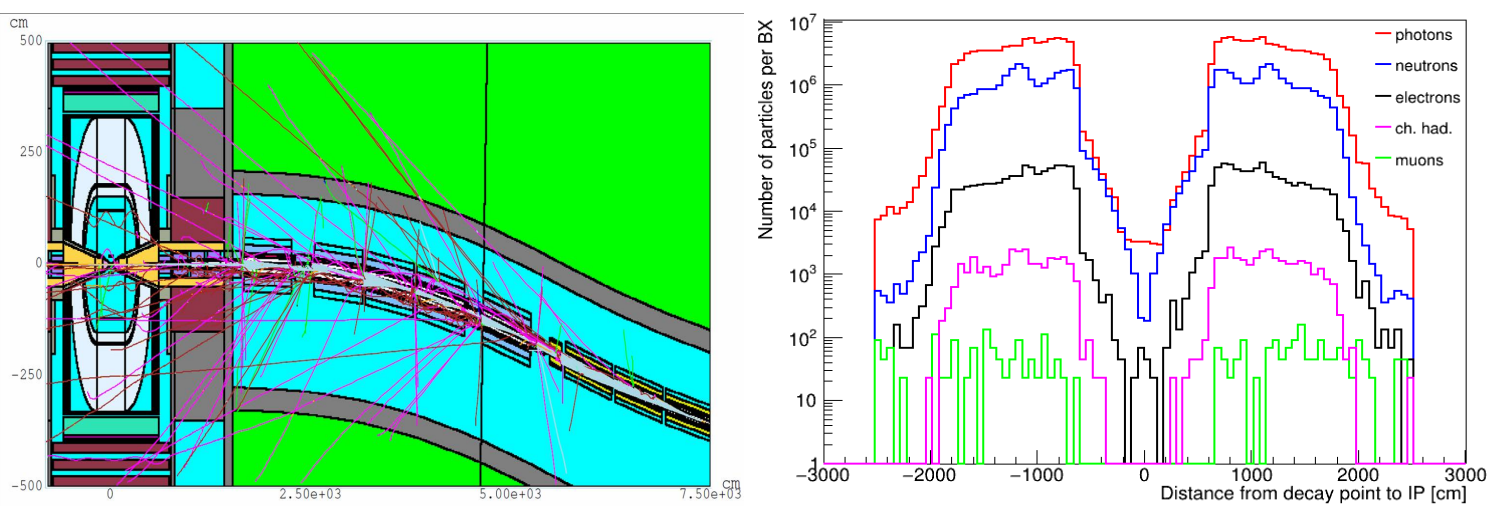

Figure 1: Left: Simulation model of the Interaction Region, the detector with the nozzles and the beaminduced background. Right: Particle composition of the beam-induced-background as a function of muon decay distance from the IP for the case of a $1.5 \mathrm{TeV}$ collider.
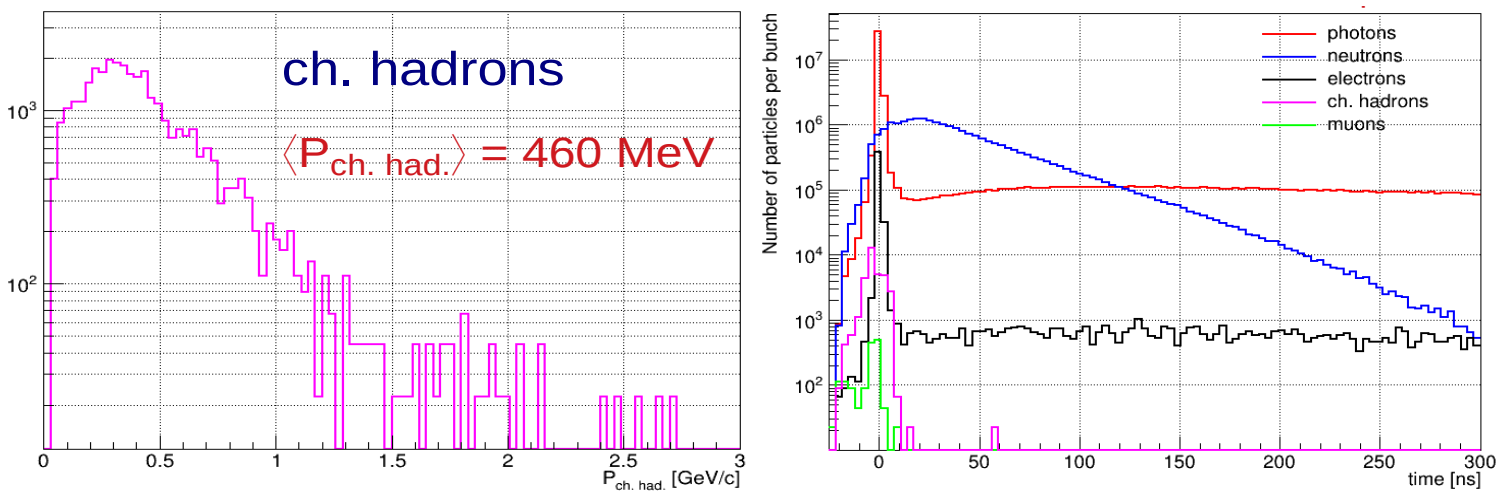

Figure 2: Left: Momentum spectra of the beam-induced background particles at the detector entry point. Right: Time of arrival of the background particles at the detector entry point with respect to the bunch crossing time.

old studies by the MAP collaboration which was designed back in 2014. The tracking system is composed by silicon pixel detectors with $20 \mu \mathrm{m}$ and $50 \mu \mathrm{m}$ pitch respectively [7], it is surrounded by a dual-readout calorimeter based on the ADRIANO design [9]. Everything is immersed in a solenoidal magnetic field of $3.57 \mathrm{~T}$. Muon detector, supposed to be in the outer region is not simulated in this study. Full simulation of the detector response is performed with a software based on the ILCRoot framework [7] and takes into account sensor efficiency and noise, saturation effects, light attenuation in crystals and other experimental effects tuned on test beam experience. The framework is used also to reconstruct physics objects: tracks and jets for the moment.

\subsection{Tracking detectors performance}

The presence of the nozzles mitigates the effects of the beam-induced background but the amount of particles is still very high, in particular close to the beam. The occupancy on the first layers of both the central and forward region of the tracker is largely reduced by using a high spatial resolution pixel detector and by applying a cut on the hits arrival time. The time resolution is assumed to be of $50 \mathrm{ps}$ for silicon pitch of about $75 \mu \mathrm{m}$ and to be of $100 \mathrm{ps}$ for pitch of about 
$200 \mu \mathrm{m}$, and the hit is retained if it is in a window of $[-3 \sigma,+4 \sigma]$. The effectiveness of the method is shown in Figure 3 on the left, where the occupancy, defined as the number of hit clusters per $\mathrm{cm}^{2}$ area, is plotted. Layers 0-4 (VTX barrel) and 13-17 (SiT barrel) correspond to the central tracker, from the closer to the more distant from the beam pipe. Other layers, VTX, SiT and FTD endcaps, correspond to the forward/backward detectors, from the closer to the more distant from the nominal interaction point. The tracking efficiency has been studie in detail, here it is shown
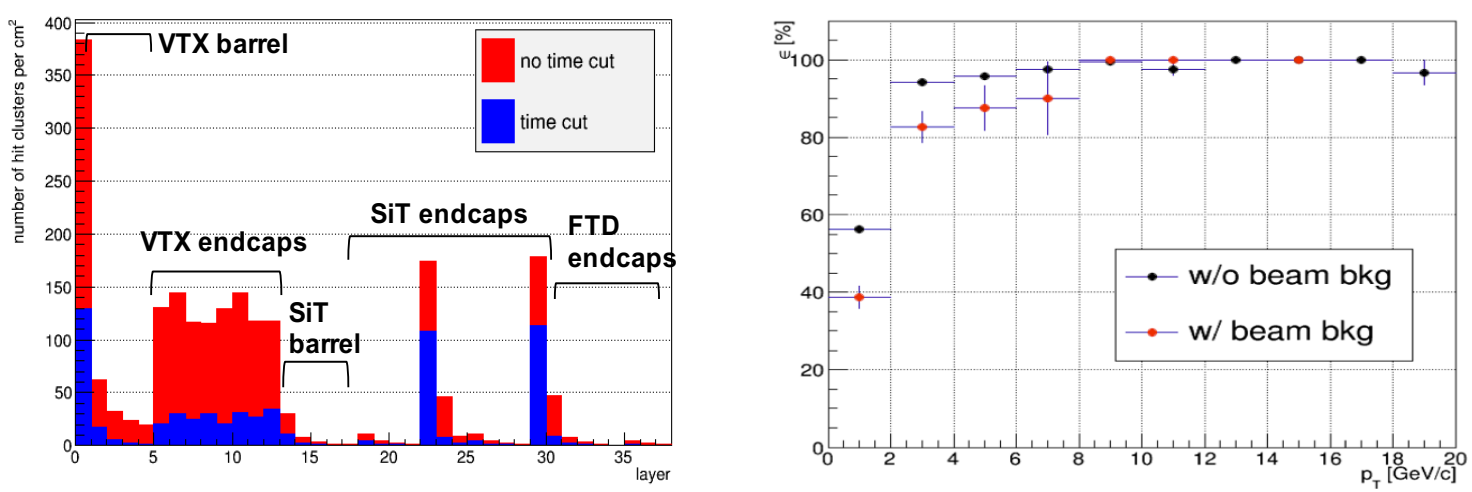

Figure 3: Left: Simulated occupancy as a function of tracker layer number before and after applying hit arrival time requirement to be in the selection window. Right: Track reconstruction efficiency for the signal events described in the text with and without the beam-induced background as function of track transverse momentum.

the result on a physics benchmark process. A sample of events $\mu^{+} \mu^{-} \rightarrow H \nu \bar{v} \rightarrow b \bar{b} \nu \bar{v}$ generated with Pythia [8] at $\sqrt{s}=1.5 \mathrm{TeV}$ is fully simulated without and with the beam-induced background. Tracks are reconstructed and the efficiency as a function of the track transverse momentum is shown in Figure 3 (right). The presence of the beam-induced background reduce the efficiency for low momentum tracks due to a limitation in the software, to recover low momentum tracks a lot of cpu time is needed at the moment.

\subsection{Calorimeter detector performance}

Jet reconstruction was not part of the original ILCRoot package, a specific algorithm to take into account the beam-induced background is implemented but it is not optimized. This background acts as an underlying event, contributing with an almost flat energy distribution in the phase space. The instrumentation of the calorimeter with a timing detector will help to reduce the amount of energy collected by each cell. As shown in Figure 4 (left) a fraction particles of the beam-induced background arrive significantly later than the prompt muons coming from the IP, therefore they can be rejected during data acquisition. This feature is not currently implemented. The algorithm used to reconstruct jets proceeds as follow:

- the calorimeter $\phi-\eta$ phase space is divided in several regions, and in each region the mean deposited energy $\bar{E}$ and the standard deviation $\sigma_{\bar{E}}$ are calculated for the beam-induced background using the simulated events; 
- signal plus beam-induced background events are analyzed, for each cluster, if $E_{c l}-\bar{E}>$ $2 \times \sigma_{\bar{E}}$ then the cluster is added to the clusters list with $E_{c l}^{\prime}=E_{c l}-\bar{E}$;

- a standard jet cone algorithm [10] with radius $R=0.5$, is used on the list of clusters with the corrected energy;

The jet reconstruction efficiency as a function of the jet transverse momentum is shown on the right in Figure 4. The maximum efficiency of $70 \%$ is obtained at high momentum with a minimum of $50 \%$ at $40 \mathrm{GeV}$. Even if the algorithm is not optimal, its performance is good enough to use it for
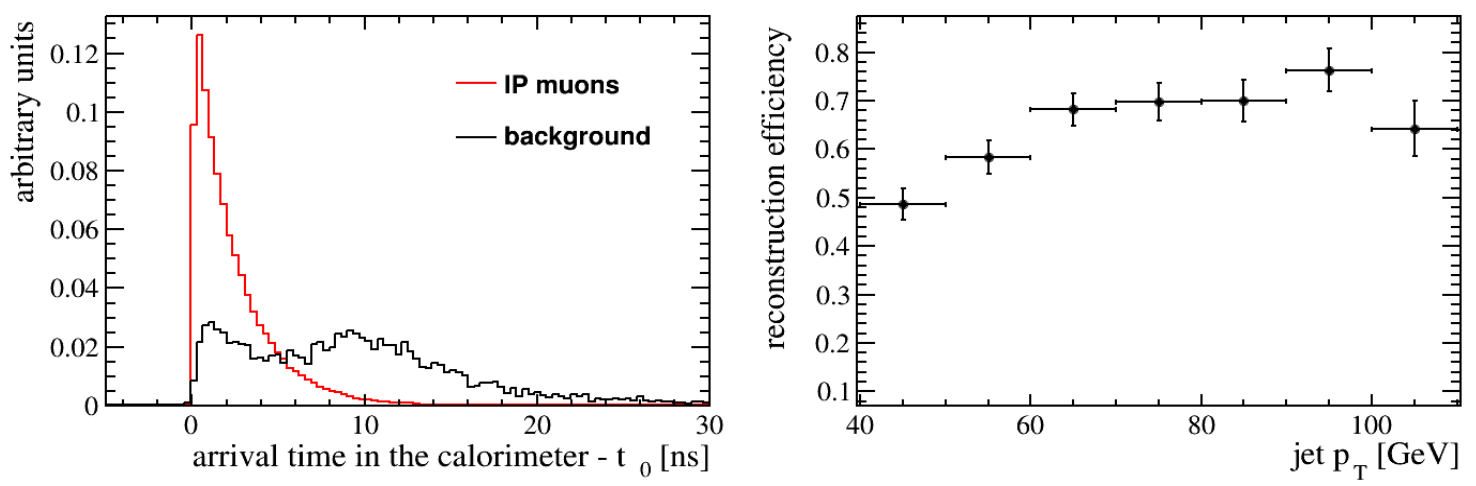

Figure 4: Left: Distribution of the arrival time at each calorimeter cell of the beam-induced background particles and prompt muons coming from IP. Right: Jets reconstruction efficiency as a function of jet transverse momentum.

physics studies. The correlation between the jet transverse momentum at truth-level in the monte carlo simulation and the reconstructed transverse momentum plotted in Figure 5 (left) is very good. Given the method used for the jet reconstruction a large energy correction is needed. In the physics studies, jets with corrected momentum are used. After the correction is applied, the jet transverse momentum momentum resolution is of about $30 \%$, almost flat on the full transverse momentum range.

Figure 5 (right) shows the two $b$-jets invariant mass when each jet is identified as a $b$-jet using the truth-level information in the fully simulated sample $\mu^{+} \mu^{-} \rightarrow H v \bar{v} \rightarrow b \bar{b} v \bar{v}$ previously described. The $b$-jet identification, (tagging), algorithm is not applied to the Higgs reconstruction. Most of the tracks belonging to a $b$-jet coming from Higgs generated $\mu^{+} \mu^{-} \rightarrow H \nu \bar{v} \rightarrow b \bar{b} v \bar{v}$ at $1.5 \mathrm{TeV}$ center-of-mass energy have transverse momenta below $2 \mathrm{GeV}$. The current tracking algorithm has a degraded efficiency at low momentum due to software configuration, which does not allow to use $b$-jet tagging on these events. Preliminary studies show that if tracks are reconstructed with good efficiency down to $400 \mathrm{MeV}$ the $b$-jet tagging based only on secondary vertex identification has an efficiency of about $60 \%$ on average.

\section{Summary and future plans}

A preliminary study of the effects of the machine-induced background on the detector performance was carried out using the MAP simulation framework and MARS15 generated backgrounds 

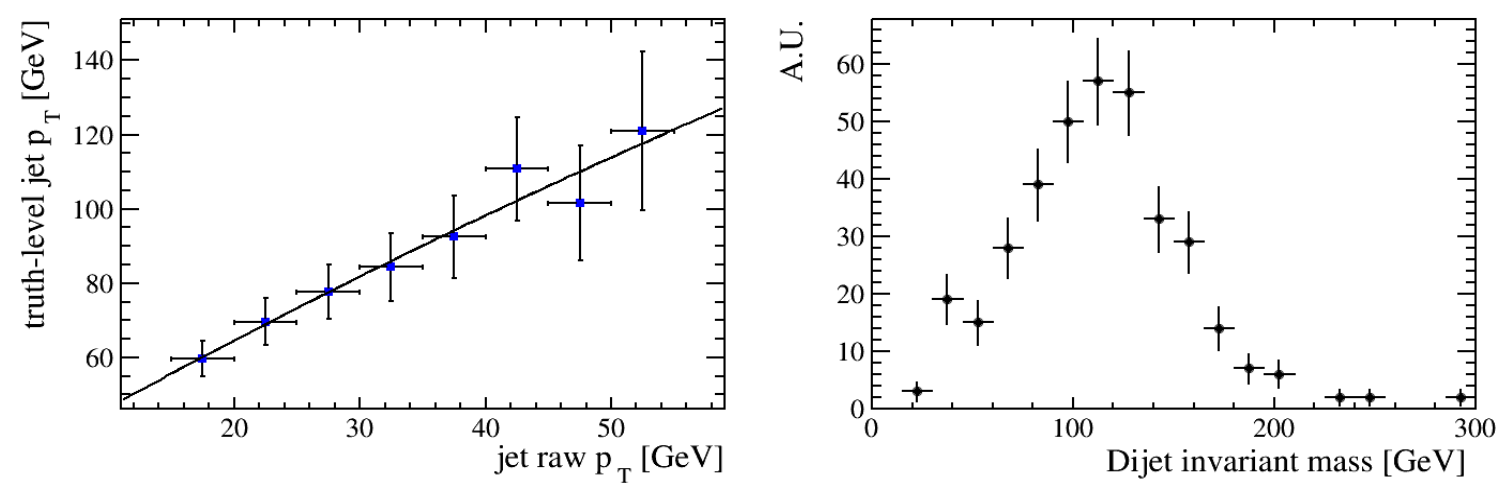

Figure 5: Left: Distribution of the jet transverse momentum at Monte Carlo truth-level as a function of the reconstructed jet transverse momentum. Right: Invariant mass of two $b-$ jets for $H \rightarrow b \bar{b}$ events.

for a muon collider with a center-of-mass energy of $1.5 \mathrm{TeV}$. Track and jet reconstruction results are very promising although the detector and the algorithms used are not optimal.

The community of high energy physics at colliders is making an effort to implement a common framework for the study of physics performance at future colliders, therefore the muon collider simulation and reconstruction algorithms are being ported to the new framework. A short term plan is to optimize the tracking detector and the track reconstruction method, to adopt a modern and performing algorithm for jet finding and for $b$-jet identification exploiting machine learning methods and to include the muon detector and reconstruction for a center-of-mass energy of $1.5 \mathrm{TeV}$. In the future the detector will be optimized also for other center-of-mass energies, including the beam-induced background.

\section{References}

[1] J. de Blas, M. Cepeda, J. D’Hondt, R. K. Ellis, C. Grojean, B. Heinemann, F. Maltoni, A. Nisati, E. Petit, R. Rattazzi, W. Verkerke, Higgs Boson Studies at Future Particle Colliders, arXiv:1905.03764.

[2] J.P. Delahaye, M. Diemoz, K. Long, B. Mansoulié, N. Pastrone, L. Rivkin, D. Schulte, A. Skrinsky, A. Wulzer, Muon Colliders, arXiv:1901.06150 [physics.acc-ph] (2019).

[3] The Muon Accelerator Program, https://map.fnal.gov/.

[4] N.V. Mokhov and S.I. Striganov, Detector Backgrounds at Muon Colliders, Phys. Procedia 37 (2012) [arXiv:1204.6721].

[5] N.V. Mokhov, S.I. Striganov, and I.S. Tropin, Reducing backgrounds in the Higgs factory muon collider detector, arXiv:1409.1939 (2014).

[6] N.V. Mokhov and C.C. James, The MARS Code System UserâǍŹs Guide Version 15 (2016), FERMILAB-FN-1058-APC (2017)

[7] V. Di Benedetto et al., A study of muon collider background rejection criteria in silicon vertex and tracker detectors, 2018 JINST 13 P09004

[8] T. SjÃústrand, S. Mrenna and P. Skands, JHEP05 (2006) 026, Comput. Phys. Comm. 178 (2008) 852.

[9] C. Gatto et al., Status of Dual-readout R\&D for a linear collider in T1015 Collaboration, arXiv:1603.00909 [physics.ins-det] (2016)

[10] G. C. Blazeyet al., hep-ex/0005012. 